\title{
On a coupled system of random and stochastic differential equations with nonlocal stochastic integral conditions
}

\author{
A. M. A. El-Sayed and Hoda A. Fouad \\ Faculty of Science, Alexandria University, Alexandria, Egypt \\ amasayed@alexu.edu.eg, hoda-rg@yahoo.com
}

\begin{abstract}
Here we are concerning with two problems of a coupled system of random and stochastic nonlinear differential equations with two coupled systems of nonlinear nonlocal random and stochastic integral conditions. The existence of solutions will be studied. The sufficient condition for the uniqueness of the solution will be given. The continuous dependence of the unique solution on the nonlocal conditions will be proved.
\end{abstract}

2010 MSC.34A12, 34A30, 34D20, 34F05, 60H10.

Key words. Stochastic processes, stochastic differential equation, coupled system, nonlocal stochastic integral conditions.

\section{Introduction}

The coupled systems of differential equations with nonlocal conditions have studied by some authors ( see for example [4]-[6] and references therein).

Let $(\Omega, \digamma, P)$ be a fixed probability space, where $\Omega$ is a sample space, $\digamma$ is a $\sigma$-algebra and $P$ is a probability measure.

Let $Z(t ; \omega)=Z(t), t \in[0, T], \omega \in \Omega$ be a second order stochastic process, i.e., $E\left(Z^{2}(t)\right)<$ $\infty, t \in[0, T]$.

Let $C=C\left([0, T], L_{2}(\Omega)\right)$ be the space of all second order stochastic processes which is mean square (m.s) continuous on $[0, T]$. The norm of $Z \in C\left([0, T], L_{2}(\Omega)\right)$ is given by

$$
\|Z\|_{C}=\sup _{t \in[0, T]}\|Z(t)\|_{2}, \quad \quad\|Z(t)\|_{2}=\sqrt{E\left(Z^{2}(t)\right)} .
$$


Let $T \geq 1$. In this paper we study the existence of solutions $(x, y) \in C\left([0, T], L_{2}(\Omega)\right)$ of the problem of the coupled system of random and stochastic differential equations

$$
\begin{aligned}
\frac{d x(t)}{d t} & =f_{1}\left(t, y\left(\phi_{1}(t)\right)\right), \quad t \in(0, T], \\
d y(t) & =f_{2}\left(t, x\left(\phi_{2}(t)\right)\right) d W(s), \quad t \in(0, T]
\end{aligned}
$$

subject to each one of the two nonlinear nonlocal stochastic integral conditions

$$
x(0)+\int_{0}^{\tau} h_{1}(s, y(s)) d W(s)=x_{0}, \quad y(0)+\int_{0}^{\eta} h_{2}(s, x(s)) d s=y_{0}
$$

and

$$
x(0)+\int_{0}^{\tau} h_{1}(s, x(s)) d W(s)=x_{0}, \quad y(0)+\int_{0}^{\eta} h_{2}(s, y(s)) d s=y_{0}
$$

where $x_{0}$ and $y_{0}$ are two second order random variables.

Let $X=C\left([0, T], L_{2}(\Omega)\right) \times C\left([0, T], L_{2}(\Omega)\right)$ be the class of all ordered pairs $(x, y), \quad x, y \in C$ with the norm

$$
\|(x, y)\|_{X}=\max \left\{\|x\|_{C},\|y\|_{C}\right\}=\max \left\{\sup _{t \in[0, T]}\|x(t)\|_{2}, \sup _{t \in[0, T]}\|y(t)\|_{2}\right\} .
$$

Let $\phi_{i}:[0, T] \rightarrow[0, T]$ be continuous functions such that $\phi_{i}(t) \leq t$ and consider the following assumptions

(A1) $f_{i}:[0, T] \times L_{2}(\Omega) \rightarrow L_{2}(\Omega), \quad i=1,2$ are measurable in $t \in[0, T]$ for all $x \in L_{2}(\Omega)$ and continuous in $x \in L_{2}(\Omega)$ for all $t \in[0, T]$. There exist two bounded measurable functions $m_{i}:[0, T] \rightarrow R$ and two positive constants $b_{i}$ such that

$$
\left\|f_{i}(t, x)\right\|_{2} \leq\left|m_{i}(t)\right|+b_{i}\|x(t)\|_{2}, \quad i=1,2 .
$$

(A2) $\quad h_{i}:[0, T] \times L_{2}(\Omega) \rightarrow L_{2}(\Omega), \quad i=1,2$ are measurable in $t \in[0, T]$ for all $x \in L_{2}(\Omega)$ and continuous in $x \in L_{2}(\Omega)$ for all $t \in[0, T]$. There exist two bounded measurable functions $k_{i}:[0, T] \rightarrow R$ and two positive constants $c_{i}$ such that

$$
\left\|h_{i}(t, x)\right\|_{2} \leq\left|k_{i}(t)\right|+c_{i}\|x(t)\|_{2}, \quad i=1,2 .
$$

(A3) $M=\max \left\{\sup _{t \in[0, T]}\left|m_{1}(t)\right|, \sup _{t \in[0, T]}\left|m_{2}(t)\right|\right\}, \quad b=\max \left\{b_{1}, b_{2}\right\}$.

(A4) $K=\max \left\{\sup _{t \in[0, T]}\left|k_{1}(t)\right|, \sup _{t \in[0, T]}\left|k_{2}(t)\right|\right\}, c=\max \left\{c_{1}, c_{2}\right\}$.

(A5) $(b+c) T<1$.

Now, integrating the two random and stochastic differential equations (1)-(2) (see [2], [3], [7]-[11]) and using the nonlocal conditions (3)and (4) the following Lemma can be proved. 
Lemma 1. The integral representations of the solutions of the nonlocal problems (1)-(2) with conditions (3) and (1) - (2) with conditions (4) are given by

$$
\begin{aligned}
& x(t)=x_{0}-\int_{0}^{\tau} h_{1}(s, y(s)) d W(s)+\int_{0}^{t} f_{1}\left(s, y\left(\phi_{1}(s)\right)\right) d s, \\
& y(t)=y_{0}-\int_{0}^{\eta} h_{2}(s, x(s)) d s+\int_{0}^{t} f_{2}\left(s, x\left(\phi_{2}(s)\right)\right) d W(s) .
\end{aligned}
$$

and

$$
\begin{aligned}
& x(t)=x_{0}-\int_{0}^{\tau} h_{1}(s, x(s)) d W(s)+\int_{0}^{t} f_{1}\left(s, y\left(\phi_{1}(s)\right)\right) d s \\
& y(t)=y_{0}-\int_{0}^{\eta} h_{2}(s, y(s)) d s+\int_{0}^{t} f_{2}\left(s, x\left(\phi_{2}(s)\right)\right) d W(s) .
\end{aligned}
$$

respectively.

\section{Solutions of the problem (1)-(3)}

Define the mapping $F(x, y)=\left(F_{1} y, F_{2} x\right)$ where $F_{1} y, F_{2} x$ are given by the following stochastic integral equations

$$
\begin{aligned}
& F_{1} y(t)=x_{0}-\int_{0}^{\tau} h_{1}(s, y(s)) d W(s)+\int_{0}^{t} f_{1}\left(s, y\left(\phi_{1}(s)\right)\right) d s \\
& F_{2} x(t)=y_{0}-\int_{0}^{\eta} h_{2}(s, x(s)) d s+\int_{0}^{t} f_{2}\left(s, x\left(\phi_{2}(s)\right)\right) d W(s) .
\end{aligned}
$$

Consider the set $Q$ such that

$$
Q=\left\{x, y \in L_{2}(\Omega),(x, y) \in X:\|(x, y)\|_{X}=\max \left\{\|x(t)\|_{2},\|y(t)\|_{2}\right\} \leq r .\right\}
$$

Now, we have the following two lemmas

Lemma 2. $F: Q \rightarrow Q$.

Proof. Let $y \in Q, \quad\|y(t)\|_{2} \leq r_{1}$, then

$$
\begin{aligned}
\left\|F_{1} y(t)\right\|_{2} & \leq\left\|x_{0}\right\|_{2}+\left\|\int_{0}^{\tau} h_{1}(s, y(s)) d W(s)\right\|_{2}+\left\|\int_{0}^{t} f_{1}\left(s, y\left(\phi_{1}(s)\right)\right) d s\right\|_{2} \\
& \leq\left\|x_{0}\right\|_{2}+\sqrt{\int_{0}^{\tau}\left\|h_{1}(s, y(s))\right\|_{2}^{2} d s}+\int_{0}^{t}\left\|f_{1}\left(s, y\left(\phi_{1}(s)\right)\right)\right\|_{2} d s \\
& \leq\left\|x_{0}\right\|_{2}++\sqrt{\int_{0}^{\tau}\left(\left|k_{1}(s)\right|+c_{1}\|y(s)\|_{2}\right)^{2}} d s+\int_{0}^{t}\left(\left|m_{1}(S)\right|+b_{1}\|y(s)\|_{2}\right) d s \\
& \leq\left\|x_{0}\right\|_{2}+\left(K+c r_{1}\right) \sqrt{T}+\left(M+b r_{1}\right) T<\left\|x_{0}\right\|_{2}+\left(K+c r_{1}\right) T+\left(M+b r_{1}\right) T=r_{1}
\end{aligned}
$$


where

$$
r_{1}=\frac{\left\|x_{0}\right\|_{2}+K T+M T}{1-(b+c) T}>0 .
$$

Let $x \in Q, \quad\|x(t)\|_{2} \leq r_{2}$, then

$$
\begin{aligned}
\left\|F_{2} x(t)\right\|_{2} & \leq\left\|y_{0}\right\|_{2}+\left\|\int_{0}^{\eta} h_{2}(s, x(s)) d s\right\|_{2}+\left\|\int_{0}^{t} f_{2}\left(s, x\left(\phi_{2}(s)\right)\right) d W(s)\right\|_{2} \\
& \leq\left\|y_{0}\right\|_{2}+\int_{0}^{\eta}\left\|h_{2}(s, x(s))\right\|_{2} d s+\sqrt{\int_{0}^{t}\left\|f_{2}\left(s, x\left(\phi_{2}(s)\right)\right)\right\|_{2}^{2} d s} \\
& \leq\left\|y_{0}\right\|_{2}+\int_{0}^{\eta}\left(\left|k_{2}(s)\right|+c_{2}\|x(s)\|_{2}\right) d s+\sqrt{\int_{0}^{t}\left(\left|m_{2}(t)\right|+b_{2}\|x\|_{2}\right)^{2} d s} \\
& \leq\left\|y_{0}\right\|_{2}+\left(K+c r_{2}\right) T+\left(M+b r_{2}\right) T<\left\|y_{0}\right\|_{2}+\left(K+c r_{2}\right) T+\left(M+b r_{2}\right) T=r_{2}
\end{aligned}
$$

where

$$
r_{2}=\frac{\left\|y_{0}\right\|_{2}+K T+M T}{1-(b+c) T}>0 .
$$

Let $r=\max \left\{r_{1}, r_{2}\right\}, \quad(x, y) \in Q$, then

$$
\begin{aligned}
\|F(x, y)\|_{X} & =\left\|\left(F_{1} y, F_{2} x\right)\right\|_{X} \\
& =\max \left\{\left\|F_{1} y(t)\right\|_{C},\left\|F_{2} x(t)\right\|_{C}\right\}<r .
\end{aligned}
$$

This proves that $F: Q \rightarrow Q$ and the class of functions $\{F(x, y)(t)\}, t \in[0, T]$ is uniformly bounded on $Q$.

Lemma 3. The class of function $\{F(x, y)(t)\}, t \in[0, T]$ is equicontinuous.

Proof. Let $x, y \in Q, \quad t_{1}, t_{2} \in[0, T]$ such that $\left|t_{2}-t_{1}\right|<\delta$, then

$$
\begin{aligned}
\left\|F_{1} y\left(t_{1}\right)-F_{1} y\left(t_{2}\right)\right\|_{2} & =\left\|\int_{0}^{t_{1}} f_{1}\left(s, y\left(\phi_{1}(s)\right)\right) d s-\int_{0}^{t_{2}} f_{1}\left(s, y\left(\phi_{1}(s)\right)\right) d s\right\|_{2} \\
& \leq \int_{t_{1}}^{t_{2}}\left\|f_{1}\left(s, y\left(\phi_{1}(s)\right)\right)\right\|_{2} \\
& \leq\left(M+b\|y\|_{C}\right)\left(t_{2}-t_{1}\right)
\end{aligned}
$$

and

$$
\begin{aligned}
\left\|F_{2} x\left(t_{1}\right)-F_{2} x\left(t_{2}\right)\right\|_{2} & =\left\|\int_{0}^{t_{1}} f_{2}\left(s, x\left(\phi_{2}(s)\right)\right) d W(s)-\int_{0}^{t_{2}} f_{2}\left(s, x\left(\phi_{2}(s)\right)\right) d W(s)\right\|_{2} \\
& \leq \sqrt{\int_{t_{1}}^{t_{2}}\left\|f_{2}\left(s, x\left(\phi_{2}(s)\right)\right)\right\|_{2}^{2} d s} \\
& \leq\left(M+b\|x\|_{C}\right) \sqrt{\left(t_{2}-t_{1}\right)}
\end{aligned}
$$


But

$$
\begin{aligned}
F\left(x\left(t_{2}\right), y\left(t_{2}\right)\right)-F\left(x\left(t_{1}\right), y\left(t_{1}\right)\right) & =\left(F_{1} y\left(t_{2}\right), F_{2} x\left(t_{2}\right)\right)-\left(F_{1} y\left(t_{1}\right), F_{2} x\left(t_{1}\right)\right) \\
& =\left(\left(F_{1} y\left(t_{2}\right)-F_{1} y\left(t_{1}\right)\right),\left(F_{2} x\left(t_{2}\right)-F_{2} x\left(t_{1}\right)\right)\right),
\end{aligned}
$$

then from (14) and (14), we can deduce the equicontinuity of the class $\{F(x, y)(t)\}, \quad t \in$ $[0, T]$ on $Q$.

\subsection{Existence Theorem}

Now, we have the following existence theorem

Theorem 1. Let the assumptions (A1) - (A5) be satisfied, then there exists at least one solution $(x, y) \in X$ of the problem $(1)-(3)$.

Proof. Let $\left(x_{n}, y_{n}\right) \in Q$ be such that $\left(x_{n}, y_{n}\right) \rightarrow(x, y) \quad$ w.p.1.

Using lemmas 1-3, then applying stochastic Lebesgue dominated convergence Theorem [1], we can obtain

$$
\begin{aligned}
\text { L.i. } m_{n \rightarrow \infty} F\left(x_{n}, y_{n}\right)= & \left(\text { L.i. } m_{n \rightarrow \infty} F_{1} y_{n}, L . i . m_{n \rightarrow \infty} F_{2} x_{n}\right) \\
= & \left(\text { L.i. } m_{n \rightarrow \infty}\left\{x_{0}-\int_{0}^{\tau} h_{1}\left(s, y_{n}(s)\right) d W(s)+\int_{0}^{t} f_{1}\left(s, y_{n}\left(\phi_{1}(s)\right)\right) d s\right\},\right. \\
& \text { L.i.m } \left.m_{n \rightarrow \infty}\left\{y_{0}-\int_{0}^{\eta} h_{2}\left(s, x_{n}(s)\right) d s+\int_{0}^{t} f_{2}\left(s, x_{n}\left(\phi_{2}(s)\right)\right) d W(s)\right\}\right) \\
= & \left(x_{0}-\int_{0}^{\tau} h_{1}\left(s, L . i . m_{n \rightarrow \infty} y_{n}(s)\right) d W(s)+\int_{0}^{t} f_{1}\left(s, L . i . m_{n \rightarrow \infty} y_{n}\left(\phi_{1}(s)\right)\right) d s,\right. \\
& \left.y_{0}-\int_{0}^{\eta} h_{2}\left(s, L . i . m_{n \rightarrow \infty} x_{n}(s)\right) d s+\int_{0}^{t} f_{2}\left(s, L . i . m_{n \rightarrow \infty} x_{n}\left(\phi_{2}(s)\right)\right) d W(s)\right) \\
= & \left(x_{0}-\int_{0}^{\tau} h_{1}(s, y(s)) d W(s)+\int_{0}^{t} f_{1}\left(s, y\left(\phi_{1}(s)\right)\right) d s,\right. \\
& \left.y_{0}-\int_{0}^{\eta} h_{2}(s, x(s)) d s+\int_{0}^{t} f_{2}\left(s, x\left(\phi_{2}(s)\right)\right) d W(s)\right) \\
= & \left(F_{1} y, F_{2} x\right)=F(x, y) .
\end{aligned}
$$

This proves that the operator $F: Q \rightarrow Q$ is continuous.

Then by Arzela-Ascoli Theorem [1], the closure of $F Q$ is a compact subset of $X$, then applying Schauder Fixed Point Theorem [1], there exists at least one solution $(x, y) \in X$ of the problem (1)-(3) such that $x, y \in C\left([0, T], L_{2}(\Omega)\right)$.

\subsection{Uniqueness Theorem}

Replace the assumptions $(A 1)$ and $(A 2)$ by $\left(A^{*} 1\right)$ and $\left(A^{*} 2\right)$ respectively such that $\left(A^{*} 1\right)$ The functions $f_{i}:[0, T] \times L_{2}(\Omega) \rightarrow L_{2}(\Omega), \quad i=1,2$ are measurable in $t \in[0, T]$ 
for all $x \in L_{2}(\Omega)$ and satisfy Lipschitz condition with respect to the second argument

$$
\left\|f_{i}(t, u)-f_{i}(t, v)\right\|_{2} \leq b\|u-v\|_{2} .
$$

$\left(A^{*} 2\right)$ The functions $h_{i}:[0, T] \times L_{2}(\Omega) \rightarrow L_{2}(\Omega), \quad i=1,2$ are measurable in $t \in[0, T]$ for all $x \in L_{2}(\Omega)$ and satisfy Lipschitz condition with respect to the second argument

$$
\left\|h_{i}(t, u)-h_{i}(t, v)\right\|_{2} \leq c\|u-v\|_{2}
$$

Remark. Let the assumptions $\left(A^{*} 1\right)$ and $\left(A^{*} 2\right)$ be satisfied, then we can get

$$
\begin{gathered}
\left\|f_{i}(t, u)\right\|_{2}-\left\|f_{i}(t, 0)\right\|_{2} \leq\left\|f_{i}(t, u)-f_{i}(t, 0)\right\|_{2} \leq b\|u\|_{2}, \\
\left\|f_{i}(t, u)\right\|_{2} \leq\left\|f_{i}(t, 0)\right\|_{2}+b\|u\|_{2} \leq M+b\|u\|_{2}
\end{gathered}
$$

and

$$
\left\|h_{i}(t, u)\right\|_{2} \leq\left\|h_{i}(t, 0)\right\|_{2}+c\|u\|_{2} \leq K+c\|u\|_{2} .
$$

Theorem 2. Let the assumptions $\left(A^{*} 1\right)-\left(A^{*} 2\right)$ and $(A 3)-(A 5)$ be satisfied, then the solution of problem (1)- (3) is unique.

Proof. Let $\left(x_{1}, y_{1}\right)$ and $\left(x_{2}, y_{2}\right)$ be two solutions of the problem (1)-(3), then

$$
\begin{aligned}
& (x(t), y(t))=\left(x_{0}-\int_{0}^{\tau} h_{1}(s, y(s)) d W(s)+\int_{0}^{t} f_{1}\left(s, y\left(\phi_{1}(s)\right)\right) d s,\right. \\
& \left.y_{0}-\int_{0}^{\eta} h_{2}(s, x(s)) d s+\int_{0}^{t} f_{2}\left(s, x\left(\phi_{2}(s)\right)\right) d W(s)\right)
\end{aligned}
$$

where

$$
\begin{aligned}
\left\|x_{1}(t)-x_{2}(t)\right\|_{2} & \leq\left\|\int_{0}^{\tau}\left[h_{1}\left(s, y_{2}(s)\right)-h_{1}\left(s, y_{1}(s)\right)\right] d W(s)\right\|_{2}+\left\|\int_{0}^{t}\left(f_{1}\left(s, y_{1}\right)-f_{1}\left(s, y_{2}\right)\right) d s\right\|_{2} \\
& \leq \sqrt{\int_{0}^{\tau} c^{2}\left\|y_{2}-y_{1}\right\|_{C}^{2} d s+T b\left\|y_{1}-y_{2}\right\|_{C} \leq T \sqrt{c}\left\|y_{1}-y_{2}\right\|_{C}+T b\left\|y_{1}-y_{2}\right\|_{C}} \\
& \leq T(b+c)\left\|y_{1}-y_{2}\right\|_{C} \\
& \leq T(b+c) \max \left\{\left\|x_{1}-x_{2}\right\|_{C},\left\|y_{1}-y_{2}\right\|_{C}\right\}
\end{aligned}
$$

and

$$
\begin{aligned}
\left\|y_{1}(t)-y_{2}(t)\right\|_{2} & \leq \int_{0}^{\eta}\left\|h_{2}\left(s, x_{2}(s)\right)-h_{2}\left(s, x_{1}(s)\right)\right\|_{2} d s+\sqrt{\left.\left.\left.\int_{0}^{t} b^{2} \| x_{1}(s)\right)-x_{2}(s)\right)\right) \|_{2}^{2} d s} \\
& \leq \sqrt{T} b\left\|x_{1}-x_{2}\right\|_{C}+c T\left\|x_{2}-x_{1}\right\|_{C} \\
& \leq T(b+c)\left\|x_{1}-x_{2}\right\|_{C}, \\
& \leq T(b+c) \max \left\{\left\|x_{1}-x_{2}\right\|_{C},\left\|y_{1}-y_{2}\right\|_{C}\right\} .
\end{aligned}
$$


Hence

$$
\begin{aligned}
\|\left(x_{1}, y_{1}\right)-\left(x_{2}, y_{2} \|_{X}\right. & =\left\|\left(x_{1}-x_{2}\right),\left(y_{1}, y_{2}\right)\right\|_{X} \\
& =\max \left\{\left\|\left(x_{1}-x_{2}\right)\right\|_{C},\left\|\left(y_{1}, y_{2}\right)\right\|_{C}\right\} \\
& \leq T(b+c) \max \left\{\left\|x_{1}-x_{2}\right\|_{C},\left\|y_{2}-y_{1}\right\|_{C}\right\} \\
& \leq T(b+c)\left\|\left(x_{1}, y_{1}\right)-\left(x_{2}, y_{2}\right)\right\|_{X} .
\end{aligned}
$$

This implies that

$$
(1-T(b+c))\left\|\left(x_{1}, y_{1}\right)-\left(x_{2}, y_{2}\right)\right\|_{X} \leq 0
$$

and

$$
\left\|\left(x_{1}, y_{1}\right)-\left(x_{2}, y_{2}\right)\right\|_{X}=0
$$

then $\left(x_{1}, y_{1}\right)=\left(x_{2}, y_{2}\right)$ and the solution of the problem (1)-(3) is unique.

\subsection{Continuous Dependence}

Theorem 3. Let the assumptions of Theorem 2 be satisfied. Then the solution (15) of the problem (1) - (3) depends continuously on the two random data $\left(x_{0}, y_{0}\right)$.

Proof. Let $(\hat{x}, \hat{y})$ be the solution of the coupled system

$$
\begin{aligned}
& \hat{x}(t)=\hat{x}_{0}-\int_{0}^{\tau} h_{1}(s, \hat{y}(s)) d W(s)+\int_{0}^{t} f_{1}\left(s, \hat{y}\left(\phi_{1}(s)\right)\right) d s \\
& \hat{y}(t)=\hat{y}_{0}-\int_{0}^{\eta} h_{2}(s, \hat{x}(s)) d s+\int_{0}^{t} f_{2}\left(s, \hat{x}\left(\phi_{2}(s)\right)\right) d W(s),
\end{aligned}
$$

such that $\left\|\left(x_{0}, y_{0}\right)-\left(\hat{x_{0}}, \hat{y_{0}}\right)\right\|_{X}<\delta_{1}$, then

$$
\begin{aligned}
\|x-\hat{x}\|_{C} & \leq\left\|x_{0}-\hat{x_{0}}\right\|_{C}+T(b+c)\|y-\hat{y}\|_{C} \\
& \leq \delta_{1}+T(b+c)\|y-\hat{y}\|_{C} \\
& \leq \delta_{1}+T(b+c) \max \left\{\|x-\hat{x}\|_{C},\|y-\hat{y}\|_{C}\right\} \\
\|y-\hat{y}\|_{C} & \leq\left\|y_{0}-\hat{y_{0}}\right\|_{C}+T(b+c)\|x-\hat{x}\|_{C}, \\
& \leq \delta_{1}+T(b+c)\|x-\hat{x}\|_{C} \\
& \leq \delta_{1}+T(b+c) \max \left\{\|x-\hat{x}\|_{C},\|y-\hat{y}\|_{C}\right\} .
\end{aligned}
$$

Then

$$
\begin{aligned}
\|(x, y)-(\hat{x}, \hat{y})\|_{X} & =\|(x-\hat{x}, y-\hat{y})\|_{X} \\
& =\max \left\{\|x-\hat{x}\|_{C},\|y-\hat{y}\|_{C}\right\} \\
& \leq \delta_{1}+T(b+c) \max \left\{\|x-\hat{x}\|_{C},\|y-\hat{y}\|_{C}\right\} \\
& \leq \delta_{1}+T(b+c)\|(x, y)-(\hat{x}, \hat{y})\|_{X} .
\end{aligned}
$$

This implies that

$$
\|(x, y)-(\hat{x}, \hat{y})\|_{X} \leq \frac{\delta_{1}}{1-T(b+c)}=\epsilon
$$


which completes the proof.

Theorem 4. The solution (15) of the problem (1)-(3) depends continuously on the two random functions $h_{1}$ and $h_{2}$.

Proof. Let $(\hat{x}, \hat{y})$ be the solutions of the coupled system

$$
\begin{aligned}
& \hat{x}(t)=x_{0}-\int_{0}^{\tau} h_{1}^{*}(s, \hat{y}(s)) d W(s)+\int_{0}^{t} f_{1}\left(s, \hat{y}\left(\phi_{1}(s)\right)\right) d s, \\
& \hat{y}(t)=y_{0}-\int_{0}^{\eta} h_{2}^{*}(s, \hat{x}(s)) d s+\int_{0}^{t} f_{2}\left(s, \hat{x}\left(\phi_{2}(s)\right)\right) d W(s)
\end{aligned}
$$

such that $\left\|h_{i}^{*}(s, .)-h(s, .)\right\|_{2} \leq \delta_{2}, \quad i=1,2$, then

$$
\begin{aligned}
& \|x(t)-\hat{x}(t)\|_{2}=\left\|\int_{0}^{\tau}\left[h_{1}^{*}(s, \hat{y}(s))-h_{1}(s, y(s))\right] d W(s)+\int_{0}^{t}\left[f_{1}\left(s, y\left(\phi_{1}(s)\right)\right)-f_{1}\left(s, \hat{y}\left(\phi_{1}(s)\right)\right)\right] d s\right\|_{2} \\
& \leq \sqrt{\int_{0}^{\tau}\left\|h_{1}^{*}(s, \hat{y}(s))-h_{1}(s, y(s))\right\|_{2}^{2} d s}+\int_{0}^{t}\left\|f_{1}\left(s, y\left(\phi_{1}(s)\right)\right)-f_{1}\left(s, \hat{y}\left(\phi_{1}(s)\right)\right)\right\|_{2} d s \\
& \leq \sqrt{\int_{0}^{\tau}\left[\left\|h_{1}^{*}(s, \hat{y}(s))-h_{1}^{*}(s, y(s))\right\|_{2}+\left\|h_{1}^{*}(s, y(s))-h_{1}(s, y(s))\right\|_{2}\right]^{2} d s} \\
& +\int_{0}^{t}\left\|f_{1}\left(s, y\left(\phi_{1}(s)\right)\right)-f_{1}\left(s, \hat{y}\left(\phi_{1}(s)\right)\right)\right\|_{2} d s \\
& \leq \sqrt{\int_{0}^{\tau}\left(c\|y(s)-\hat{y}(s)\|_{2}+\delta_{2}\right)^{2} d s}+\int_{0}^{t} b\|y(s)-\hat{y}(s)\|_{2} d s \\
& \leq(c \sqrt{T}+b T)\|y-\hat{y}\|_{C}+\delta_{2} \sqrt{T} \\
& \leq T(b+c) \max \left\{\|x-\hat{x}\|_{C},\|y-\hat{y}\|_{C}\right\}+\delta_{2} T
\end{aligned}
$$

Similarly we can get

$$
\begin{aligned}
\|y(t)-\hat{y}(t)\|_{2} & =\left\|\int_{0}^{\eta}\left[h_{2}^{*}(s, \hat{x}(s))-h_{2}(s, x(s))\right] d s+\int_{0}^{t}\left[f_{2}\left(s, x\left(\phi_{2}(s)\right)\right)-f_{2}\left(s, \hat{x}\left(\phi_{2}(s)\right)\right)\right] d W(s)\right\|_{2} \\
& \leq(c T+b \sqrt{T})\|x-\hat{x}\|_{C}+\delta_{2} T \\
& \leq T(b+c)\|x-\hat{x}\|_{C}+\delta_{2} T \\
& \leq T(b+c) \max \left\{\|x-\hat{x}\|_{C},\|y-\hat{y}\|_{C}\right\}+\delta_{2} T \\
& \leq T(b+c) \max \left\{\|x-\hat{x}\|_{C},\|y-\hat{y}\|_{C}\right\}+\delta_{2} T
\end{aligned}
$$

Now

$$
\begin{aligned}
\|(x, y)-(\hat{x}, \hat{y})\|_{X} & =\max \left\{\|x-\hat{x}\|_{C},\|y-\hat{y}\|_{C}\right. \\
& \leq T(b+c) \max \left\{\left(\|x-\hat{x}\|_{C},\|y-\hat{y}\|_{C}\right\}+\delta_{2} T\right. \\
& \leq T(c+b)\|(x, y)-(\hat{x}, \hat{y})\|_{X}+\delta_{2} T .
\end{aligned}
$$


This implies that

$$
\|(x, y)-(\hat{x}, \hat{y})\|_{X} \leq \frac{\delta_{2} T}{1-T(b+c)}=\epsilon
$$

which completes the proof.

\section{Solutions of the problem (1)-(2) and (4)}

Define the mapping $L(x, y)=\left(L_{1} x, L_{2} y\right)$ where $L_{1} x, L_{2} y$ are given by the following stochastic integral equations

$$
\begin{aligned}
& L_{1} x(t)=x_{0}-\int_{0}^{\tau} h_{1}(s, x(s)) d W(s)+\int_{0}^{t} f_{1}\left(s, y\left(\phi_{1}(s)\right)\right) d s \\
& L_{2} y(t)=y_{0}-\int_{0}^{\eta} h_{2}(s, y(s)) d s+\int_{0}^{t} f_{2}\left(s, x\left(\phi_{2}(s)\right)\right) d W(s) .
\end{aligned}
$$

Lemma 4. $\quad L: Q \rightarrow Q$.

Proof. Let $x, y \in Q$, then we obtain

$$
\begin{aligned}
\left\|L_{1} x(t)\right\|_{2} & \leq\left\|x_{0}\right\|_{2}+\left\|\int_{0}^{\tau} h_{1}(s, x(s)) d W(s)\right\|_{2}+\left\|\int_{0}^{t} f_{1}\left(s, y\left(\phi_{1}(s)\right)\right) d s\right\|_{2} \\
& \leq\left\|x_{0}\right\|_{2}+\sqrt{\int_{0}^{\tau}\left\|h_{1}(s, x(s))\right\|_{2}^{2} d s+\int_{0}^{t}\left\|f_{1}\left(s, y\left(\phi_{1}(s)\right)\right)\right\|_{2} d s} \\
& \leq\left\|x_{0}\right\|_{2}++\sqrt{\int_{0}^{\tau}\left(\left|k_{1}(s)\right|+c_{1}\|x(s)\|_{2}\right)^{2}} \int_{0}^{t}\left(\left|m_{1}(s)\right|+b_{1}\|y(s)\|_{2}\right) d s \\
& \left.\leq\left\|x_{0}\right\|_{2}+K \sqrt{T}+M T+c \sqrt{T}\|x\|_{C}+b T\|y\|_{C}\right) \\
& \leq\left\|x_{0}\right\|_{2}+\left\|y_{0}\right\|_{2}+(K+M) T+2 r T(b+c)
\end{aligned}
$$

and

$$
\begin{aligned}
\left\|L_{2} y(t)\right\|_{2} & \leq\left\|y_{0}\right\|_{2}+\left\|\int_{0}^{\eta} h_{2}(s, y(s)) d s\right\|_{2}\left\|\int_{0}^{t} f_{2}\left(s, x\left(\phi_{2}(s)\right)\right) d W(s)\right\|_{2} \\
& \leq\left\|y_{0}\right\|_{2}=\int_{0}^{\eta}\left\|h_{2}(s, y(s))\right\|_{2} d s+\sqrt{\int_{0}^{t}\left\|f_{2}\left(s, x\left(\phi_{2}(s)\right)\right)\right\|_{2}^{2} d s} \\
& \leq\left\|y_{0}\right\|_{2}+\int_{0}^{\eta}\left(\left|k_{2}(s)\right|+c_{2}\|y(s)\|_{2}\right) d s+\sqrt{\int_{0}^{t}\left(\left|m_{2}(t)\right|+b_{2}\|x\|_{2}\right)^{2} d s} \\
& \leq\left\|y_{0}\right\|_{2}+K T+M \sqrt{T}+c T\|y\|_{C}+b \sqrt{T}\|x\|_{C} \\
& \leq\left\|y_{0}\right\|_{2}+(K+M) T+T(b+c)\|y\|_{C}+T(b+c)\|x\|_{C} \\
& \leq\left\|x_{0}\right\|_{2}+\left\|y_{0}\right\|_{2}+(K+M) T+2 r T(b+c) .
\end{aligned}
$$


This implies that

$$
\begin{aligned}
\|L(x, y)\|_{X} & =\left\|\left(L_{1} x, L_{2} y\right)\right\|_{X} \\
& =\max \left\{\left\|L_{1} x(t)\right\|_{C},\left\|L_{2} y(t)\right\|_{C}\right\} \\
& \leq\left\|x_{0}\right\|_{2}+\left\|y_{0}\right\|_{2}+(K+M) T+2 r T(b+c)=r
\end{aligned}
$$

where

$$
r=\frac{\left\|x_{0}\right\|_{2}+\left\|y_{0}\right\|_{2}++(K+M) T}{1-T(b+c)},
$$

then the class $\{L(x, y)\}$ is uniformly bounded and $L(x, y): Q \rightarrow Q$.

Lemma 5. The class of function $\{L(x, y)(t)\}, t \in[0, T]$ is equicontinuous.

Proof. Let $x, y \in Q, \quad t_{1}, t_{2} \in[0, T]$ such that $\left|t_{2}-t_{1}\right|<\delta$, then

$$
\begin{aligned}
\left\|L_{1} x\left(t_{2}\right)-L_{1} y\left(t_{1}\right)\right\|_{2} & =\left\|\int_{0}^{t_{2}} f_{1}\left(s, y\left(\phi_{1}(s)\right)\right) d s-\int_{0}^{t_{1}} f_{1}\left(s, y\left(\phi_{1}(s)\right)\right) d s\right\|_{2} \\
& \leq \int_{t_{1}}^{t_{2}}\left\|f_{1}\left(s, y\left(\phi_{1}(s)\right)\right)\right\|_{2} d s \\
& \leq\left(M+b\|y\|_{C}\right)\left(t_{2}-t_{1}\right)
\end{aligned}
$$

and

$$
\begin{aligned}
\left\|L_{2} x\left(t_{2}\right)-L_{2} x\left(t_{1}\right)\right\|_{2} & =\left\|\int_{0}^{t_{2}} f_{2}\left(s, x\left(\phi_{2}(s)\right)\right) d W(s)-\int_{0}^{t_{1}} f_{2}\left(s, x\left(\phi_{2}(s)\right)\right) d W(s)\right\|_{2} \\
& \leq \sqrt{\int_{t_{1}}^{t_{2}}\left\|f_{2}\left(s, x\left(\phi_{2}(s)\right)\right)\right\|_{2}^{2} d s} \\
& \leq\left(M+b\|x\|_{C}\right) \sqrt{\left(t_{2}-t_{1}\right)}
\end{aligned}
$$

But

$$
\begin{aligned}
L\left(x\left(t_{2}\right), y\left(t_{2}\right)\right)-L\left(x\left(t_{1}\right), y\left(t_{1}\right)\right) & =\left(L_{1} x\left(t_{2}\right), L_{2} y\left(t_{2}\right)\right)-\left(L_{1} x\left(t_{1}\right), L_{2} y\left(t_{1}\right)\right) \\
& =\left(\left(L_{1} x\left(t_{2}\right)-L_{1} x\left(t_{1}\right)\right),\left(L_{2} y\left(t_{2}\right)-L_{2} y\left(t_{1}\right)\right)\right),
\end{aligned}
$$

then from (18) and (19), we deduce the equicontinuity of the class $\{L(x, y)(t)\}$ on $Q$.

\subsection{Existence Theorem}

Now, we have the following existence theorem

Theorem 5. Let the assumptions (A1) - (A5) be satisfied, then there exists at least one solution $(x, y) \in X$ of the problem (1)-(2) and (4).

Proof. Let $\left\{\left(x_{n}, y_{n}\right)\right\} \in Q$ be such that $\left(x_{n}, y_{n}\right) \rightarrow(x, y) \quad$ w.p.1. 
Using lemmas 1-3, then applying stochastic Lebesgue dominated convergence Theorem [1], we can obtain

$$
\begin{aligned}
\text { L.i. } m_{n \rightarrow \infty} L\left(x_{n}, y_{n}\right)= & \left(\text { L.i. } m_{n \rightarrow \infty} L_{1} x_{n}, L . i . m_{n \rightarrow \infty} L_{2} y_{n}\right) \\
= & \left(\text { L.i. } m_{n \rightarrow \infty}\left\{x_{0}-\int_{0}^{\tau} h_{1}\left(s, x_{n}(s)\right) d W(s)+\int_{0}^{t} f_{1}\left(s, y_{n}\left(\phi_{1}(s)\right)\right) d s\right\},\right. \\
& \text { L.i. } \left.m_{n \rightarrow \infty}\left\{y_{0}-\int_{0}^{\eta} h_{2}\left(s, y_{n}(s)\right) d s+\int_{0}^{t} f_{2}\left(s, x_{n}\left(\phi_{2}(s)\right)\right) d W(s)\right\}\right) \\
= & \left(x_{0}-\int_{0}^{\tau} h_{1}\left(s, L . i . m_{n \rightarrow \infty} x_{n}(s)\right) d W(s)+\int_{0}^{t} f_{1}\left(s, L . i . m_{n \rightarrow \infty} y_{n}\left(\phi_{1}(s)\right)\right) d s,\right. \\
& \left.y_{0}-\int_{0}^{\eta} h_{2}\left(s, L . i . m_{n \rightarrow \infty} y_{n}(s)\right) d s+\int_{0}^{t} f_{2}\left(s, L . i . m_{n \rightarrow \infty} x_{n}\left(\phi_{2}(s)\right)\right) d W(s)\right) \\
= & \left(x_{0}-\int_{0}^{\tau} h_{1}(s, x(s)) d W(s)+\int_{0}^{t} f_{1}\left(s, y\left(\phi_{1}(s)\right)\right) d s,\right. \\
& \left.y_{0}-\int_{0}^{\eta} h_{2}(s, y(s)) d s+\int_{0}^{t} f_{2}\left(s, x\left(\phi_{2}(s)\right)\right) d W(s)\right) \\
= & \left(L_{1} x, L_{2} y\right)=L(x, y) .
\end{aligned}
$$

This proves that the operator $L: Q \rightarrow Q$ is continuous.

Then by the Arzela-Ascoli Theorem [1], the closure of $L Q$ is a compact subset of $X$, then applying Schauder Fixed Point Theorem [1], there exists at least one solution $(x, y) \in X$ of the problem (1)-(2) and (4) such that $x, y \in C\left([0, T], L_{2}(\Omega)\right)$.

\subsection{Uniqueness Theorem}

Theorem 6. Let the assumptions $\left(A^{*} 1\right)-\left(A^{*} 2\right)$ and $(A 3)-(A 5)$ be satisfied then the solution of problem (1)- (2) and (4) is unique.

Proof. Let $\left(x_{1}, y_{1}\right)$ and $\left(x_{2}, y_{2}\right)$ be two solutions of the problem (1)-(2) and(4) on the form

$$
\begin{aligned}
& (x(t), y(t))=\left(x_{0}-\int_{0}^{\tau} h_{1}(s, x(s)) d W(s)+\int_{0}^{t} f_{1}\left(s, y\left(\phi_{1}(s)\right)\right) d s,\right. \\
& \left.y_{0}-\int_{0}^{\eta} h_{2}(s, y(s)) d s+\int_{0}^{t} f_{2}\left(s, x\left(\phi_{2}(s)\right)\right) d W(s)\right),
\end{aligned}
$$

then we can get

$$
\begin{aligned}
\left\|x_{1}(t)-x_{2}(t)\right\|_{2} & \leq c \sqrt{T}\left\|x_{1}-x_{2}\right\|_{C}+b T\left\|y_{1}-y_{2}\right\|_{C}<c T\left\|x_{1}-x_{2}\right\|_{C}+b T\left\|y_{1}-y_{2}\right\|_{C} \\
& \leq(b+c) T\left\|x_{1}-x_{2}\right\|_{C}+(b+c) T\left\|y_{1}-y_{2}\right\|_{C} \\
& \leq(b+c) T \max \left\{\left\|x_{1}-x_{2}\right\|_{C}, \quad\left\|y_{1}-y_{2}\right\|_{C}\right\} .
\end{aligned}
$$

Similarly, we can obtain

$$
\left\|y_{1}(t)-y_{2}(t)\right\|_{2} \leq(b+c) T \max \left\{\left\|x_{1}-x_{2}\right\|_{C}, \quad\left\|y_{1}-y_{2}\right\|_{C}\right\}
$$


Hence from (21) and (22)

$$
\begin{aligned}
\left\|\left(x_{1}, y_{1}\right)-\left(x_{2}, y_{2}\right)\right\|_{X} & =\left\|\left(x_{1}-x_{2}\right),\left(y_{1}-y_{2}\right)\right\|_{X} \\
& \leq \max \left\{\left\|x_{1}-x_{2}\right\| C,\left\|y_{1}-y_{2}\right\|_{C}\right\} \\
& \leq(b+c) T \max \left\{\left\|x_{1}-x_{2}\right\|_{C}, \quad\left\|y_{1}-y_{2}\right\|_{C}\right\}
\end{aligned}
$$

This implies that

$$
(1-(b+c) T)\left\|\left(x_{1}, y_{1}\right)-\left(x_{2}, y_{2}\right)\right\|_{X} \leq 0
$$

Then

$$
\left\|\left(x_{1}, y_{1}\right)-\left(x_{2}, y_{2}\right)\right\|_{X}=0
$$

and $\left(x_{1}, y_{1}\right)=\left(x_{2}, y_{2}\right)$ which proves that the solution of the problem (1)-(2) and (4) is unique.

\subsection{Continuous Dependence}

Theorem 7. The solution (15) of the problem (1) - (2) and (4) depends continuously on the two random data $\left(x_{0}, y_{0}\right)$.

Proof. Let $(\hat{x}, \hat{y})$ be the solution of the coupled system

$$
\begin{aligned}
& \hat{x}(t)=\hat{x}_{0}-\int_{0}^{\tau} h_{1}(s, \hat{x}(s)) d W(s)+\int_{0}^{t} f_{1}\left(s, \hat{y}\left(\phi_{1}(s)\right)\right) d s \\
& \hat{y}(t)=\hat{y}_{0}-\int_{0}^{\eta} h_{2}(s, \hat{y}(s)) d s+\int_{0}^{t} f_{2}\left(s, \hat{x}\left(\phi_{2}(s)\right)\right) d W(s),
\end{aligned}
$$

such that $\left\|\left(x_{0}, y_{0}\right)-\left(\hat{x_{0}}, \hat{y}_{0}\right)\right\|_{X}<\delta_{3}$. Then we have

$$
\begin{aligned}
x(t)-\hat{x}(t) & =x_{0}-\hat{x_{0}}-\int_{0}^{\tau}\left[h_{1}(s, \hat{x}(s))-h_{1}(s, x(s))\right] d W(s) \\
& +\int_{0}^{t}\left[f_{1}\left(s, y\left(\phi_{1}(s)\right)\right)-f_{1}\left(s, \hat{y}\left(\phi_{1}(s)\right)\right)\right] d s
\end{aligned}
$$

and

$$
\begin{aligned}
\|x(t)-\hat{x}(t)\|_{2} & \leq\left\|x_{0}-\hat{x_{0}}\right\|_{C}+c \sqrt{T}\|x-\hat{x}\|_{C}+b T\|y-\hat{y}\|_{C} \\
& \leq\left\|x_{0}-\hat{x_{0}}\right\|_{C}+c T\|x-\hat{x}\|_{C}+b T\|y-\hat{y}\|_{C} \\
& \leq\left\|x_{0}-\hat{x_{0}}\right\|_{2}+c \operatorname{Tmax}\left\{\|x-\hat{x}\|_{C},\|y-\hat{y}\|_{C}\right\}+b \operatorname{Tmax}\left\{\|x-\hat{x}\|_{C},\|y-\hat{y}\|_{C}\right\} \\
& \leq \max \left\{\left\|x_{0}-\hat{x_{0}}\right\|_{2},\left\|y_{0}-\hat{y_{0}}\right\|_{2}\right\}+(b+c) \operatorname{Tmax}\left\{\|x-\hat{x}\|_{C},\|y-\hat{y}\|_{C}\right\} .
\end{aligned}
$$

By the same way we can get

$$
\|y(t)-\hat{y}(t)\|_{2} \leq \max \left\{\left\|x_{0}-\hat{x_{0}}\right\|_{2},\left\|y_{0}-\hat{y_{0}}\right\|_{2}\right\}+(b+c) \operatorname{Tmax}\left\{\|x-\hat{x}\|_{C},\|y-\hat{y}\|_{C}\right\}
$$


and

$$
\begin{aligned}
\|(x, y)-(\hat{x}, \hat{y})\|_{X} & =\max \left\{\|\left(x-\hat{x}\left\|_{C},\right\|\left(y-\hat{y} \|_{C}\right\}\right.\right. \\
& \leq \max \left\{\left\|x_{0}-\hat{x_{0}}\right\|_{2},\left\|y_{0}-\hat{y_{0}}\right\|_{2}\right\}+(b+c) \operatorname{Tmax}\left\{\|x-\hat{x}\|_{C},\|y-\hat{y}\|_{C}\right\} \\
& \leq \delta_{3}+(b+c) \operatorname{Tmax}\left\{\|x-\hat{x}\|_{C},\|y-\hat{y}\|_{C}\right\}
\end{aligned}
$$

which give our result

$$
\|(x, y)-(\hat{x}, \hat{y})\|_{X} \leq \frac{\delta_{3}}{1-T(b+c)}=\epsilon
$$

and completes the proof.

Theorem 8. The solution (15) of the problem (1)-(2) and (4) depends continuously on the two random functions $h_{1}$ and $h_{2}$.

Proof. Let $(\hat{x}, \hat{y})$ be the solutions of the coupled system of stochastic integral equations (1)-(2) and (4) such that

$$
\begin{aligned}
& \hat{x}(t)=x_{0}-\int_{0}^{\tau} h_{1}^{*}(s, \hat{x}(s)) d W(s)+\int_{0}^{t} f_{1}\left(s, \hat{y}\left(\phi_{1}(s)\right)\right) d s \\
& \hat{y}(t)=y_{0}-\int_{0}^{\eta} h_{2}^{*}(s, \hat{y}(s)) d s+\int_{0}^{t} f_{2}\left(s, \hat{x}\left(\phi_{2}(s)\right)\right) d W(s) .
\end{aligned}
$$

Let $\left\|h_{i}^{*}(t, u(t))-h(t, u(t))\right\|_{2} \leq \delta_{4}, \quad i=1,2$ then

$$
\begin{aligned}
& \|x(t)-\hat{x}(t)\|_{2}=\left\|\int_{0}^{\tau}\left[h_{1}^{*}(s, \hat{x}(s))-h_{1}(s, x(s))\right] d W(s)+\int_{0}^{t}\left[f_{1}\left(s, y\left(\phi_{1}(s)\right)\right)-f_{1}\left(s, \hat{y}\left(\phi_{1}(s)\right)\right)\right] d s\right\|_{2} \\
& \leq \sqrt{\int_{0}^{\tau}\left\|h_{1}^{*}(s, \hat{x}(s))-h_{1}(s, x(s))\right\|_{2}^{2} d s}+\int_{0}^{t}\left\|f_{1}\left(s, y\left(\phi_{1}(s)\right)\right)-f_{1}\left(s, \hat{y}\left(\phi_{1}(s)\right)\right)\right\|_{2} d s \\
& \leq \sqrt{\int_{0}^{\tau}\left[\left\|h_{1}^{*}(s, \hat{x}(s))-h_{1}^{*}(s, x(s))\right\|_{2}+\left\|h_{1}^{*}(s, x(s))-h_{1}(s, x(s))\right\|_{2}\right]^{2} d s} \\
& +\int_{0}^{t}\left\|f_{1}\left(s, y\left(\phi_{1}(s)\right)\right)-f_{1}\left(s, \hat{y}\left(\phi_{1}(s)\right)\right)\right\|_{2} d s \\
& \leq \sqrt{\int_{0}^{\tau}\left(c\|x(s)-\hat{x}(s)\|_{2}+\delta_{4}\right)^{2} d s}+\int_{0}^{t} b\|y(s)-\hat{y}(s)\|_{2} d s \\
& \left.\leq c \sqrt{T}\|x-\hat{x}\|_{C}+b T\|y-\hat{y}\|_{C}+\delta_{4} \sqrt{T}\right) \\
& \leq c T\|x-\hat{x}\|_{C}+b T\|y-\hat{y}\|_{C}+\delta_{4} T \text {. } \\
& \leq c T \max \left\{\|x-\hat{x}\|_{C},\|y-\hat{y}\|_{C}\right\}+b T \max \left\{\|x-\hat{x}\|_{C},\|y-\hat{y}\|_{C}\right\}+\delta_{4} T \\
& \leq(b+c) T \max \left\{\|x-\hat{x}\|_{C},\|y-\hat{y}\|_{C}\right\}+\delta_{4} T \text {. }
\end{aligned}
$$

Similarly we can get

$$
\|y-\hat{y}\|_{C} \leq(b+c) T \max \left\{\|x-\hat{x}\|_{C},\|y-\hat{y}\|_{C}\right\}+\delta_{4} T
$$


and

$\|(x, y)-(\hat{x}, \hat{y})\|_{X}=\max \left\{\|x-\hat{x}\|_{C},\|y-\hat{y}\|_{C}\right\} \leq(b+c) T \max \left\{\|x-\hat{x}\|_{C},\|y-\hat{y}\|_{C}\right\}+\delta_{4} T$.

This implies that

$$
\|(x, y)-(\hat{x}, \hat{y})\|_{X} \leq \frac{\delta_{4} T}{1-T(b+c)}=\epsilon
$$

which is completes the proof.

\section{Conclusions}

Here, we proved the existence of solutions of a coupled system of random and stochastic nonlinear differential equations with coupled nonlocal random and stochastic nonlinear integral conditions. The sufficient condition for the uniqueness of the solution have been given. The continuous dependence of the unique solution have been studied.

\section{References}

[1] R. F. Curtain and A. J. Pritchard, Functional Analysis in Modern Applied Mathematics, Academic press, 1977.

[2] A.M.A. El-Sayed, On the stochastic fractional calculus operators, Journal of Fractional Calculus and Applications, 6(1), (2015) 101-109.

[3] A.M.A. El-Sayed, M.A. El-Tawil, M.S.M. Saif, F.M. Hafez, The mean square RiemannLiouville stochastic fractional derivative and stochastic fractional order differential equation, Math. Sci. Res. j. 9 no.6, (2005)142-150.

[4] A. M. A. El-Sayed and H. H. G. Hashem, A coupled System of integral equations in reflexive Banach spaces, Acta Mathematica Scientia, 32B(5), (2012) 2021-2028.

[5] A. M. A. El-Sayed, F. Gaafar and M. El-Gendy, Continuous dependence of the solution of Ito stochastic differential equation with nonlocal conditions, Applied Mathematical Sciences 10, (2016) 1971-1982.

[6] A. M. A. El-Sayed, F. Gaafar and M. El-Gendy, Continuous dependence of the solution of random fractional-order differential equation with nonlocal condition, Fractional diff. calculus, 7(1) (2017), $135 ? 149$.

[7] F.M. Hafez, A.M.A. El-Sayed, M.A. El-Tawil, On a stochastic fractional calculus, Fractional Calculus and Applied Analysis, 4, no. 1, (2001) 81-90.

[8] F.M. Hafez, The Fractional calculus for some stochastic processes, Stochastic Analysis and Applications, 22, no. 2,(2004) 507-523. 
[9] T.T. Soong, Random Differential Equations in Science and Engineering, Academic Press, New Yourk (1973).

[10] E. Wong, Stochastic Processes, Informations and Dynamical Systems, McGraw-Hill, New York (1971).

[11] E. Wong, Introduction to Random Processes, Springer-Verlag, Berlin (1980). 